\title{
COPD Care Bundle in Emergency Department Observation Unit Reduces Emergency Department Revisits
}

\author{
Muhammad A Zafar, Timothy M Loftus, Jack P Palmer, Michael Phillips, Jonathan Ko, \\ Steven R Ward, Madeline Foertsch, Amber Dalhover, Matthew E Doers, Eric W Mueller, \\ Evaline A Alessandrini, and Ralph J Panos
}

\begin{abstract}
BACKGROUND: COPD exacerbations lead to accelerated decline in lung function, poor quality of life, and increased mortality and cost. Emergency department (ED) observation units provide short-term care to reduce hospitalizations and cost. Strategies to improve outcomes in ED observation units following COPD exacerbations are needed. We sought to reduce 30-d ED revisits for COPD exacerbations managed in ED observation units through implementation of a COPD care bundle. The study setting was an 800-bed, academic, safety-net hospital with 700 annual ED encounters for COPD exacerbations. Among those discharged from ED observation unit, the 30-d all-cause ED revisit rate (ie, the outcome measure) was 49\% (baseline period: August 2014 through September 2016). METHODS: All patients admitted to the ED observation unit with COPD exacerbations were included. A multidisciplinary team implemented the COPD bundle using iterative plan-do-study-act cycles with a goal adherence of $90 \%$ (process measure). The bundle, adopted from our inpatient program, was developed using care-delivery failures and unmet subject needs. It included 5 components: appropriate inhaler regimen, 30-d inhaler supply, education on devices available after discharge, standardized discharge instructions, and a scheduled 15-d appointment. We used statistical process-control charts for process and outcome measures. To compare subject characteristics and process features, we sampled consecutive patients from the baseline $(n=50)$ and postbundle $(n=83)$ period over 5 -month and 7-month intervals, respectively. Comparisons were made using $t$ tests and chi-square tests with $P<.05$ significance. RESULTS: During baseline and postbundle periods, 410 and 165 subjects were admitted to the ED observation unit, respectively. After iterative plan-do-study-act cycles, bundle adherence reached $90 \%$ in 6 months, and the 30-d ED revisit rate declined from $49 \%$ to $30 \%(P=.003)$ with a system shift on statistical process-control charts. There was no difference in hospitalization rate from ED observation unit $(45 \%$ vs $51 \%, P=.16)$. Subject characteristics were similar in the baseline and postbundle periods. CONCLUSIONS: Reliable adherence to a COPD care bundle reduced 30-d ED revisits among those treated in the ED observation unit. Key words: COPD; quality improvement; emergency department; patient care bundles; observation units; clinical decision units. [Respir Care 2020;65(1):1-10. (C) 2020 Daedalus Enterprises]
\end{abstract}

\section{Introduction}

COPD exacerbations led to more than 1.5 million emergency department (ED) visits and 700,000 hospitalizations

Drs Zafar and Panos are affiliated with the Division of Pulmonary and Critical Care Medicine, Department of Internal Medicine, University of Cincinnati College of Medicine, Cincinnati, Ohio. Drs Loftus and Palmer and Mr Ward are affiliated with the Department of Emergency in the United States in $2010 .^{1}$ Strategies to minimize COPD-related ED visits and hospitalizations are desperately needed to improve overall disease course, quality of life, mortality and cost. ${ }^{2-7}$ In the United States, many states

Medicine, University of Cincinnati College of Medicine, Cincinnati,
Ohio. Mr Phillips and Mr Ko are affiliated with the Department of
Respiratory Therapy, University of Cincinnati Medical Center, Cincinnati, Ohio. Drs Foertsch, Dalhover, and Mueller are affiliated 
have enrolled in the Centers for Medicare and Medicaid Services Value-Based Purchasing program, which incentivizes hospitals to reduce health care costs for COPD episodes and to improve outcomes. ${ }^{8}$ This program encourages hospitals to adopt best practices and to improve care-delivery models for these patients. Since the inclusion of COPD in the Hospital Readmission Reduction Program in 2014, much effort has focused on reducing inpatient readmissions. ${ }^{9}$ However, these initiatives do not consider revisits of patients who are discharged from the ED and ED observa-

\section{See the Related Editorial on Page 127}

tion units, thus missing opportunities for improving care.

Among patients with COPD exacerbations presenting to the ED, 37-63\% are admitted to the hospital on initial presentation. ${ }^{10-14}$ Those patients who are discharged have a high ED revisit rate within $30 \mathrm{~d} .{ }^{11}$ Further, almost $50 \%$ of serious adverse events, such as early readmission and death, occur within $14 \mathrm{~d}$ after ED discharge..$^{13}$ Many hospitals operate ED observation units, where patients are treated for 24-48 $\mathrm{h}$ to determine the need for hospitalization and to reduce hospitalizations and cost. Patients with non-lifethreatening COPD exacerbations who are admitted to ED observation units represent this vulnerable population who are managed in a monitored setting to avoid hospitalization. Effective strategies to reduce ED revisits in this high-risk population are lacking.

Despite known therapies that improve care for COPD exacerbations, hospitals struggle to implement these

with the Department of Pharmacy, University of Cincinnati Medical Center, Cincinnati, Ohio. Dr Doers is affiliated with the Department of Internal Medicine, University of Cincinnati College of Medicine, Cincinnati, Ohio. Dr Alessandrini is affiliated with the Department of Pediatrics, University of Cincinnati College of Medicine, Cincinnati, Ohio. Dr Panos is affiliated with the Department of Medicine, Veterans Affairs Medical Center Cincinnati, Cincinnati, Ohio. Dr Loftus is also affiliated with the Department of Emergency Medicine, Northwestern University Feinberg School of Medicine, Chicago, Illinois.

Dr Loftus presented a version of this paper at the 2018 Society for Academic Emergency Medicine meeting, held May 15-18, 2018, in Indianapolis, Indiana.

The authors have disclosed no conflicts of interest.

Supplementary material related to this paper is available at http://www. rcjournal.com.

Correspondence: Muhammad A Zafar MD MSc, Division of Pulmonary and Critical Care Medicine, Department of Internal Medicine, University of Cincinnati College of Medicine, 231 Albert Sabin Way, ML 0564, Cincinnati, OH 45267. E-mail: zafarmd@ucmail.uc.edu.

DOI: $10.4187 /$ respcare. 07088

\section{QUICK LOOK}

\section{Current knowledge}

Interventions to prevent readmissions and emergency department (ED) revisits after COPD exacerbation are urgently needed to improve outcomes and reduce costs. ED observation units provide short-term monitored care in an effort to improve safety and reduce preventable hospitalizations. Effective interventions that improve COPD outcomes in the ED observation unit are lacking. Use of COPD care bundles among hospitalized patients have been shown to reduce readmissions.

\section{What this paper contributes to our knowledge}

Reliable implementation of a COPD care bundle in the ED observation unit reduced the rate of 30-d ED revisits by $38.6 \%$ among subjects with COPD exacerbations. The 5 bundle elements were selected based on local care-delivery failures and unmet patient needs. Use of improvement science methods and a team-based approach were needed for system redesign to incorporate COPD bundle delivery in routine care.

strategies in routine care delivery. ${ }^{15-17}$ Variation among hospitals contributes to a significant proportion of differences in utilization and outcomes. ${ }^{18,19}$ In our previous work, we identified system-level failures and unmet patient needs as potential contributors to COPD readmissions. We designed and implemented a COPD care bundle to mitigate these failures, which led to a $35 \%$ reduction in 30-d allcause readmissions. ${ }^{20}$ Despite an impressive reduction in readmissions of hospitalized patients with COPD exacerbations, our ED revisit rates of patients discharged from the ED observation unit remained high. Effectiveness of COPD care bundles in ED observation unit settings has not been tested. ${ }^{21}$ In this quasi-experimental study, we tested our hypothesis that reliable implementation of a COPD care bundle in the ED observation unit for patients with COPD exacerbations would reduce all-cause COPD ED revisits.

\section{Methods}

This was a quasi-experimental study assessing the before-and-after effect of COPD care bundle implementation on the 30-d all-cause ED revisit rate in subjects with COPD exacerbations managed in the ED observation unit. This quality improvement project was approved by University of Cincinnati Institutional Review Board (Study \#2016-7298) and was designated as "not human subject research" because the aim was to improve outcomes and to deliver best practice care reliably. 


\section{COPD CARE BundLE IN THE EMERGENCy DEPARTMENT}

This study was performed in an 800-bed, urban, academic, safety-net, regional referral center treating 75,000 ED patients per year, approximately 700 of whom have COPD exacerbations. Almost 22\% of patients with COPD exacerbations presenting to the ED are admitted to the ED observation unit after initial treatment in the ED. The ED observation unit has 16 beds that are utilized for patients with non-life-threatening acute conditions. A final disposition decision for discharge to home versus hospitalization is made at the end of a 24-36-h stay in the ED observation unit based on clinical assessment.

The patients in the ED observation unit are managed by an advanced practice provider (ie, either a physician assistant or nurse practitioner) and an emergency medicine physician 24 hours a day. Two respiratory therapists cover the ED and ED observation units at all times. A clinical pharmacist is available from 8:00 AM to midnight and supports all ED services, including the ED observation unit. The outpatient pharmacy supplies discharge medications from 8:00 AM to 4:00 PM. A care coordinator and community health worker facilitate discharge and follow-up during regular business hours. This institution utilizes the Epic electronic medical record (EMR) system (Epic Systems, Verona, Wisconsin).

During the baseline period (August 2014 through September 2016), among all ED COPD exacerbation encounters, $410(22 \%)$ were admitted to the ED observation unit. Of these patients, 185 (45.1\%) were hospitalized for further care at the end of their observation stay, and 225 (54.9\%) were discharged. The 30-d all-cause revisit rate among patients discharged from the ED observation unit prior to implementation of the COPD bundle was $49 \%$.

\section{Subjects}

We included all subjects with COPD exacerbations who were admitted to the ED observation unit. Subjects who left against medical advice or those with a primary diagnosis other than COPD exacerbation (eg, acute coronary syndrome, pulmonary embolism, pneumonia) were excluded from the analysis. COPD revisit was defined as any unplanned revisit to the ED within 30-d of discharge from ED observation unit. An index ED observation stay is one with a primary diagnosis of COPD exacerbation or a primary diagnosis of respiratory failure with COPD as a secondary diagnosis. ${ }^{9}$

Subject variables included demographics (age, gender, body mass index), COPD severity and comorbidities (ie, percent of predicted $\mathrm{FEV}_{1}$; Charlson comorbidity index ${ }^{25}$; presence of congestive heart failure; smoking status; use of home oxygen; home COPD medications prescribed; hospitalization in the last year, the last $90 \mathrm{~d}$, or the last month; ED visit in the last year, the last $90 \mathrm{~d}$, or the last month), COPD exacerbation severity (ie, vitals at ED presentation and at $3 \mathrm{~h}$ after ED presentation, white blood cell count, hemoglobin, blood urea nitrogen, blood $\mathrm{pH}$, and $\mathrm{P}_{\mathrm{aO}_{2}}$ in blood), and care delivery variables (ie, time to first dose of steroids from ED presentation, hospitalization rate from ED observation unit).

To assess whether change in outcomes was influenced by factors other than the COPD care bundle, we studied subject characteristics and care delivery variables before, during, and after bundle implementation. We retrospectively sampled 5 months of consecutive COPD exacerbation subjects $(n=50)$ who were admitted to the ED observation unit before the project initiation. Because the baseline period from August 2014 through September 2016 demonstrated a stable system with random (ie, common cause) variation only, we hypothesized that a review of 50 consecutive subjects was an appropriate representation of the system. Subject data after care bundle implementation were collected prospectively for the next 7 months. We collected data for 83 consecutive subjects during the postbundle period prospectively for comparison with subjects from the baseline period.

\section{COPD Care Bundle}

The COPD care bundle was developed by a multidisciplinary team as part of an inpatient COPD readmission reduction project. ${ }^{20}$ The bundle was designed after a comprehensive analysis of local care delivery failures identified by retrospective review of medical records of COPD subjects who were readmitted, identification of unmet patient needs that potentially contributed to readmissions through patient interviews, and recognition of known best practices published in literature. The leading failures identified were inadequate medication regimen, lack of follow-up appointments, late follow-up appointments, lack of standard emergency plan, confusion about inhalers due to different brands and devices leading to inappropriate inhaler use. ${ }^{20}$ The bundle components were limited to 5 to focus on high-yield opportunities. The process of bundle development and explanation of bundle component selection has been described previously. ${ }^{20}$ Each bundle component is evidence-based, applicable to all COPD patients, and was deemed highyield for local context. The bundle components were designed to provide an appropriate COPD medication regimen, ${ }^{22}$ a 30-d medication supply of insurance-compatible inhalers, ${ }^{23}$ personalized inhaler education, ${ }^{24}$ standardized discharge instructions, ${ }^{24}$ and a primary care follow-up appointment scheduled within $15 \mathrm{~d}$.

\section{Project Team}

A multidisciplinary team was formed to facilitate the implementation of a COPD care bundle in the ED observation unit. The team was led by a pulmonologist trained in improvement science and the medical director of the ED. Other members were 2 advanced practice providers, 2 ED pharmacists, an outpatient pharmacist, 2 RTs, a care 


\section{COPD CARE BundLE IN THE EMERGENCy DEPARTMENT}

coordinator, and a community health worker. Additional members were included on as-needed basis, such as EMR support staff, hospital information technology support, and ED physicians.

\section{Measures}

We defined each applicable component of the COPD care bundle as an opportunity. The process measure was adherence to the COPD care bundle (ie, \% of bundle components completed out of all available opportunities). The primary outcome measure was the rate of 30-d all-cause ED revisits among subjects discharged from ED observation unit after COPD exacerbation. The secondary outcome measure was the rate of 30-d ED revisits based on the number of bundle components received out of all available opportunities. The balancing (counter) measure was the rate of hospitalization from the ED observation unit.

\section{Implementation of COPD Care Bundle}

We utilized the Model for Improvement ${ }^{26}$ for transforming the care-delivery system in the ED observation unit to deliver all components of the bundle within a 24-h period for subjects with COPD exacerbation. The specific goal of the project was to increase adherence to the COPD care bundle components to $90 \%$ within 6 months. In our first multidisciplinary team meeting, the existing process was mapped, and the COPD bundle delivery process from the inpatient setting was reviewed. ${ }^{20}$ Differences in inpatient and ED observation unit settings were identified, and a proposed process map was formed for bundle component delivery in the ED observation unit. Multiple, iterative, plan-do-study-act (PDSA) testing cycles were performed.

The PDSA cycles optimized the process of each team member's role and their interactions with other members. New steps in the process were added to accommodate the unique needs of the ED observation unit setting that differed from the in-patient setting. These included the early identification of subjects likely to be discharged home, the time-sensitive nature of the ED observation unit where the typical stay is $\leq 24-36 \mathrm{~h}$, the geographic distribution of patients in one area, and increased care transitions and hand-offs due to shiftwork of team members. Real-time feedback was obtained from front-line staff and patients during testing of new processes. Successful interventions were implemented and supported by EMR changes to achieve higher reliability and sustainability. Higher reliability interventions were designed for key processes involved in COPD bundle completion. ${ }^{27}$

\section{Data Collection and Monitoring}

To obtain baseline data, all patients admitted to the ED observation unit with a diagnosis of COPD exacerbation during the 26 months prior to study initiation were identified in a retrospective EMR chart review using International Classification of Diseases, 9th Revision (ICD-9), and ICD10 codes (see the supplementary materials at http://www. rcjournal.com). ${ }^{9}$ Data elements included the total COPD exacerbation admissions to the ED observation unit, hospitalization rate from the ED observation unit, and the rate of 30-d all-cause ED revisits for those who were discharged from the ED observation unit. As the project started, monthly data reports were generated for these measures. Subject medical records of those who were discharged to home from the ED observation unit were individually reviewed to assess the number of bundle components completed per subject (process measure). This included data on all 5 bundle components as found in medical record notes, flowsheets, and future appointments. After $30 \mathrm{~d}$, subject medical records were reviewed again to assess whether the subject had an ED visit or direct hospitalization within $30 \mathrm{~d}$ of discharge. Process and outcome measures were shared transparently with the project team on a routine, monthly basis.

\section{Statistical Analysis}

We performed an interrupted time-series analysis using statistical process control (SPC) methods. ${ }^{28,29}$ We used annotated control charts ( $P$-charts) for outcome and process measures using Microsoft Excel 2013 (Microsoft, Redmond, Washington). Due to high month-to-month variation in the number of subjects who were admitted to the ED observation unit for COPD exacerbations, we instead used time series of every 10 consecutive subjects discharged from the ED observation unit for the revisit rate (outcome measure). This was done to eliminate the effect of outcome variation due to sample size in each month. A cycle of $n=10$ was deemed sufficient given the baseline rate of $\sim 50 \% .^{30}$ The initial control limits for the outcome measure were calculated using data from the baseline period. For the process measure, we used a cycle of every 5 consecutive subjects to assess adherence to the COPD care bundle. Each subject had up to 5 opportunities depending upon the applicable bundle components. Every 5 consecutive subjects would allow up to 25 potential opportunities, which was deemed sufficient for SPC to reflect true variations of a newly implemented process. ${ }^{30}$ Because the process measure evaluated the implementation of a novel care-delivery model, we developed control limits after the implementation was complete to assess whether the process remained in statistical control. We used standard criteria for special cause variation and system shifts to determine whether observed changes were due to a special assignable cause. A system shift was defined as $\geq 8$ consecutive measurements above or below the mean. New control limits were calculated if a system shift was observed. Subject characteristic variables, balancing measure, and outcome 
Table 1. Characteristics of Subjects Admitted to ED Observation Unit With COPD Exacerbation During the Sampling Period

\begin{tabular}{|c|c|c|c|c|}
\hline Variable & Total $(N=133)$ & Prebundle $(n=50)$ & Postbundle $(n=83)$ & $P$ \\
\hline Age, y & $60 \pm 9.8$ & $60.1 \pm 10$ & $59.9 \pm 9.7$ & .90 \\
\hline Gender, female & $36 \%$ & $46 \%$ & $30 \%$ & .09 \\
\hline Body mass index, $\mathrm{kg} / \mathrm{m}^{2}$ & $29.7 \pm 8.2$ & $28.1 \pm 8.8$ & $29 \pm 7.8$ & .57 \\
\hline $\mathrm{FEV}_{1}, \mathrm{~L}$ & $1.19 \pm 0.63$ & $1.3 \pm 0.6(n=33)$ & $1.1 \pm 0.6(n=62)$ & .25 \\
\hline $\mathrm{FEV}_{1} \%$ predicted & $45.8 \pm 16.3$ & $51.4 \pm 15(n=33)$ & $42.8 \pm 16(n=62)$ & .01 \\
\hline Home oxygen use & $27 \%$ & $28 \%$ & $26.5 \%$ & .38 \\
\hline Charlson score & $3.88 \pm 2.2$ & $4.5 \pm 2.3$ & $3.4 \pm 2$ & .006 \\
\hline Reduced ejection fraction $<50 \%$ on echocardiogram & $12.37 \%(n=97)$ & $14.2 \%(n=42)$ & $10.9 \%(n=55)$ & .61 \\
\hline LABA-ICS use & $82 \%$ & $60 \%$ & $90 \%$ & .004 \\
\hline LAMA use & $75.9 \%$ & $74 \%$ & $77 \%$ & .68 \\
\hline Past intubation & $17.2 \%$ & $14 \%$ & $19.2 \%$ & .44 \\
\hline ED visit in last $1 \mathrm{y}$ & $85.7 \%$ & $88 \%$ & $84.3 \%$ & .61 \\
\hline ED visit in last $3 \mathrm{mo}$ & $63.1 \%$ & $72 \%$ & $57.8 \%$ & .13 \\
\hline ED visit in last 1 mo & $37.6 \%$ & $40 \%$ & $36 \%$ & .71 \\
\hline Hospitalization in last $1 \mathrm{y}$ & $57.8 \%$ & $68 \%$ & $51 \%$ & .073 \\
\hline Hospitalization in last $3 \mathrm{mo}$ & $25.5 \%$ & $32 \%$ & $21.7 \%$ & .22 \\
\hline Hospitalization in last $1 \mathrm{mo}$ & $7.5 \%$ & $4 \%$ & $9.6 \%$ & .31 \\
\hline Heart rate at $0 \mathrm{~min}$ & $96.9 \pm 18.7$ & $96 \pm 17.7$ & $97.4 \pm 19.3$ & .68 \\
\hline Breathing frequency at $0 \mathrm{~min}$ & $20.3 \pm 4.6$ & $19.9 \pm 4.3$ & $20.5 \pm 4.7$ & .43 \\
\hline Temp at $0 \mathrm{~min}$ & $98.3 \pm 0.5$ & $98.2 \pm 0.5$ & $98.3 \pm 0.5$ & .37 \\
\hline $\mathrm{S}_{\mathrm{pO}_{2}}$ at $0 \mathrm{~min}$ & $94.1 \pm 8.7$ & $94.5 \pm 3.7$ & $93.9 \pm 10.6$ & .62 \\
\hline Supplemental $\mathrm{O}_{2}$ need at $0 \mathrm{~min}$ & $33 \%$ & $32 \%$ & $33.7 \%$ & .83 \\
\hline Heart rate at $3 \mathrm{~h}$ & $94.1 \pm 15.4$ & $94.3 \pm 15.7$ & $94 \pm 15.2$ & .90 \\
\hline Breathing frequency at $3 \mathrm{~h}$ & $19.7 \pm 4.1$ & $19.8 \pm 3.6$ & $19.6 \pm 4.5$ & .85 \\
\hline Temperature at $3 \mathrm{~h}$ & $98.2 \pm 0.4$ & $98.3 \pm 0.5$ & $98.2 \pm 0.4$ & .48 \\
\hline $\mathrm{S}_{\mathrm{pO}_{2}}$ at $3 \mathrm{~h}$ & $94.9 \pm 8.5$ & $94.9 \pm 2$ & $94.9 \pm 10.7$ & .99 \\
\hline Supplemental $\mathrm{O}_{2}$ needed at $3 \mathrm{~h}$ & $47.3 \%$ & $56 \%$ & $42.1 \%$ & .12 \\
\hline $\mathrm{pH}$ & $7.36 \pm 0.04$ & $7.37 \pm 0.03(n=40)$ & $7.36 \pm 0.04(n=77)$ & .09 \\
\hline $\mathrm{P}_{\mathrm{aCO}_{2}}, \mathrm{~mm} \mathrm{Hg}$ & $52 \pm 9.5$ & $50.4 \pm 8.2(n=40)$ & $53.4 \pm 10.1(n=77)$ & .08 \\
\hline White blood cell count & $8.88 \pm 3.4$ & $8.6 \pm 3.6$ & $9.1 \pm 3.3$ & .38 \\
\hline Hemoglobin & $13.5 \pm 1.6$ & $13.4 \pm 1.8$ & $13.5 \pm 1.5$ & .80 \\
\hline Blood urea nitrogen & $14.7 \pm 12.3$ & $13.3 \pm 5.8$ & $15.6 \pm 14.8$ & .22 \\
\hline Creatinine & $0.95 \pm 0.67$ & $0.91 \pm 0.2$ & $0.98 \pm 0.8$ & .44 \\
\hline Time to first steroids, min & $202 \pm 264$ & $143 \pm 204$ & $237 \pm 289$ & .031 \\
\hline Final disposition & $\begin{array}{l}46.6 \% \text { hospitalized } \\
53.3 \% \text { home discharge }\end{array}$ & $\begin{array}{l}44 \% \text { hospitalized } \\
56 \% \text { home discharge }\end{array}$ & $\begin{array}{l}48 \% \text { hospitalized } \\
52 \% \text { home discharge }\end{array}$ & .72 \\
\hline \multicolumn{5}{|c|}{$\begin{array}{l}\text { Data are presented as mean } \pm \text { SD for continuous variables and as percent prevalence for categorical variables. For continuous variables, } \mathrm{t} \text { test was used; for categorical variables, the } \chi^{2} \text { test was used. } \\
\mathrm{LABA}=\text { long-acting } \beta \text {-adrenergic agonist } \\
\text { ICS }=\text { inhaled corticosteroid } \\
\text { LAMA = long-acting muscarinic antagonist } \\
\mathrm{ED}=\text { emergency department } \\
\mathrm{P}_{\mathrm{aCO}_{2}=\mathrm{P}_{\mathrm{aCO}} \text { in blood }}\end{array}$} \\
\hline
\end{tabular}

measure were compared using unpaired Student $t$ tests (for continuous variables) and chi-square tests (for categorical variables) using SPSS 21 (SPSS, Chicago, Illinois), considering $P<.05$ as significant.

\section{Results}

\section{Baseline Performance}

Baseline data were measured in 410 admissions to the ED observation unit for COPD exacerbations during a 26- month period. At the end of the stay in the ED observation unit, 185 (45.1\%) subjects were hospitalized and 225 (54.9\%) were discharged to home. Among the subjects who were discharged, 110 (48.9\%) had an all-cause ED revisit within $30 \mathrm{~d}$ of discharge.

\section{Subject Characteristics}

Overall, subjects had a mean \pm SD age of $60 \pm 9.8 \mathrm{y}$, $\mathrm{FEV}_{1} 1.19 \pm 0.63 \mathrm{~L}, \mathrm{FEV}_{1} \%$ predicted of $45.8 \pm 16.3$, and were predominantly male $(64 \%)$. The detailed characteristics 


\section{COPD CARE BUNDLE IN THE EMERGENCY DEPARTMENT}

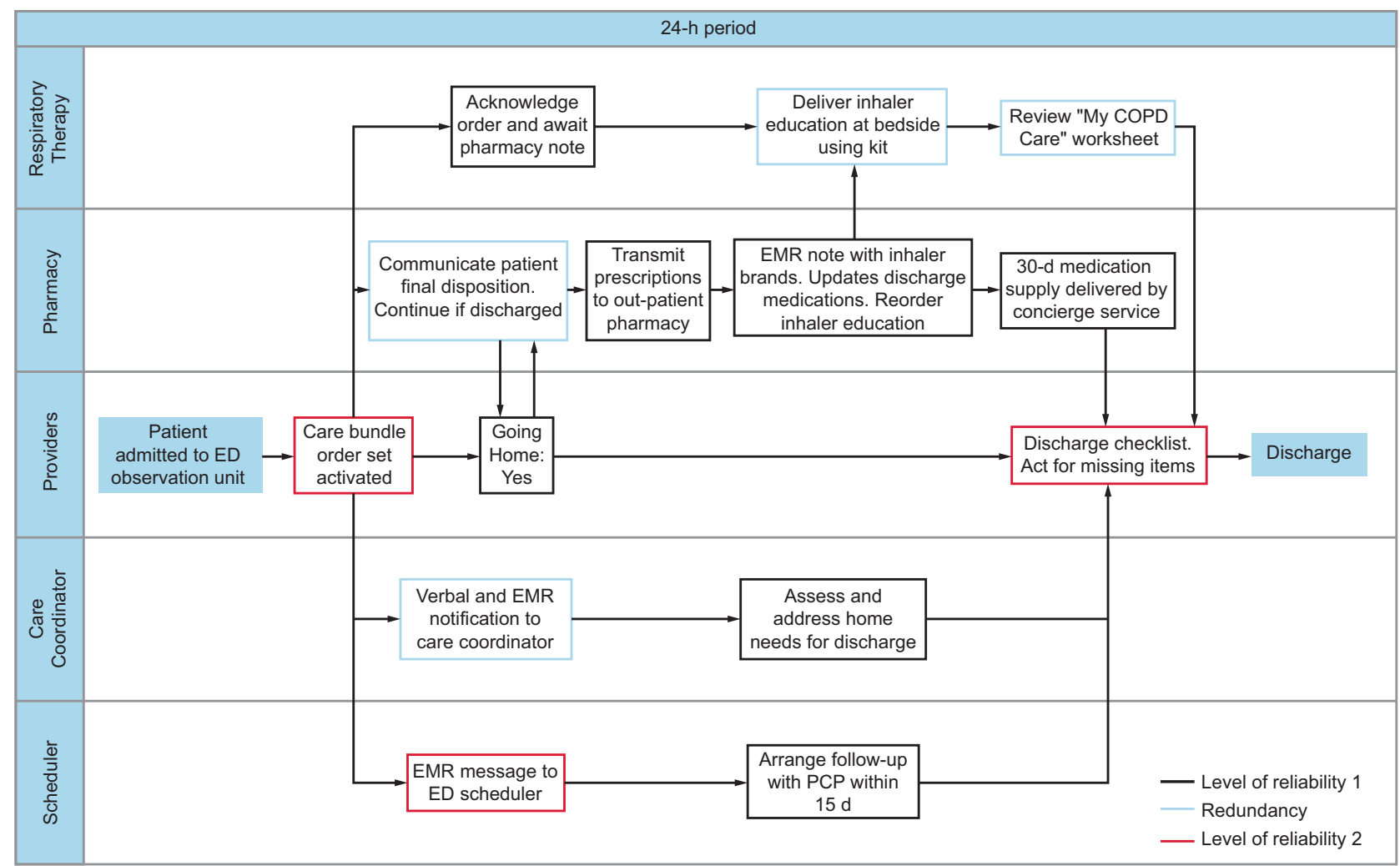

Fig. 1. COPD care bundle delivery process in ED-Obs. All team members (providers, respiratory therapists, pharmacists, care coordinators, and schedulers) have a clearly defined role in the process. Each step in the process has a known level of reliability (ie, successful completion/total number of opportunities) based on how the step is executed. The process is deliberately designed to have higher reliability (level 2) at the beginning (activation of bundle) and at the end (checklist for bundle completion). Other key steps that are time-sensitive have trigger redundancies to ensure timely completion of the overall process. A decision node is placed early in the process so that downstream actions are only taken if the patient is likely to be discharged to home from ED-Obs, thus avoiding wasteful actions if patient will be hospitalized. ED-Obs = emergency department observation unit; $d / c=$ discharge; EMR = electronic medical record; $R x=$ pharmacy; $f / u=$ follow-up; $P C P=$ primary care provider.

of subjects sampled from the baseline period (50 consecutive subjects prior to bundle intervention) and the postbundle period (83 consecutive subjects) are provided in Table 1. In comparison to the baseline group, the postbundle group had a lower Charlson comorbidity score (4.5 vs 3.4), lower $\mathrm{FEV}_{1} \%$ predicted (51.4\% vs $42.8 \%$ ), greater use of long-acting B agonist/inhaled corticosteroid inhaler (LABA/ICS) (90\% vs 60\%), and a longer time to first steroid dose in the ED (237 min vs $143 \mathrm{~min}$ ). All other variables were similar in both groups (Table 1).

\section{Implementation and Adherence to COPD Bundle (Process Measure)}

The COPD care bundle delivery was a de novo process for the ED observation unit. In the first phase of PDSA cycles, the role of each individual team member (physicians and advanced practice providers, ED and out-patient pharmacists, RTs, care coordinator, and community health worker) was optimized. In the next phase of PDSA cycles, team interactions were tested by reviewing a single patient per day and learning from each encounter. A total of 16 PDSA cycles were performed. The team process and workflow were modified iteratively until a final process, with well-defined roles and responsibilities, was developed after 3 revisions (Fig. 1). The process was then spread to all days of the week for every COPD exacerbation encounter in the ED observation unit. The interventions included common mental model of workflow, staff education and training, visual cues to facilitate optimum behavior, standard location and availability of appropriate material (see the supplementary materials at http://www. rcjournal.com), development of standard operating procedures, well-defined expectations, and changes in the EMR.

To increase and sustain high reliability (ie, rate of failure-free occurrence) of critical steps involved in the COPD care bundle delivery, we modified EMR documentation templates and added redundancy in some processes. EMR changes included implementation and utilization of a COPD care bundle order set that was adapted from the previously implemented in-patient order set, ${ }^{20}$ 


\section{COPD CARe Bundle in the EMERGENCy DePartment}

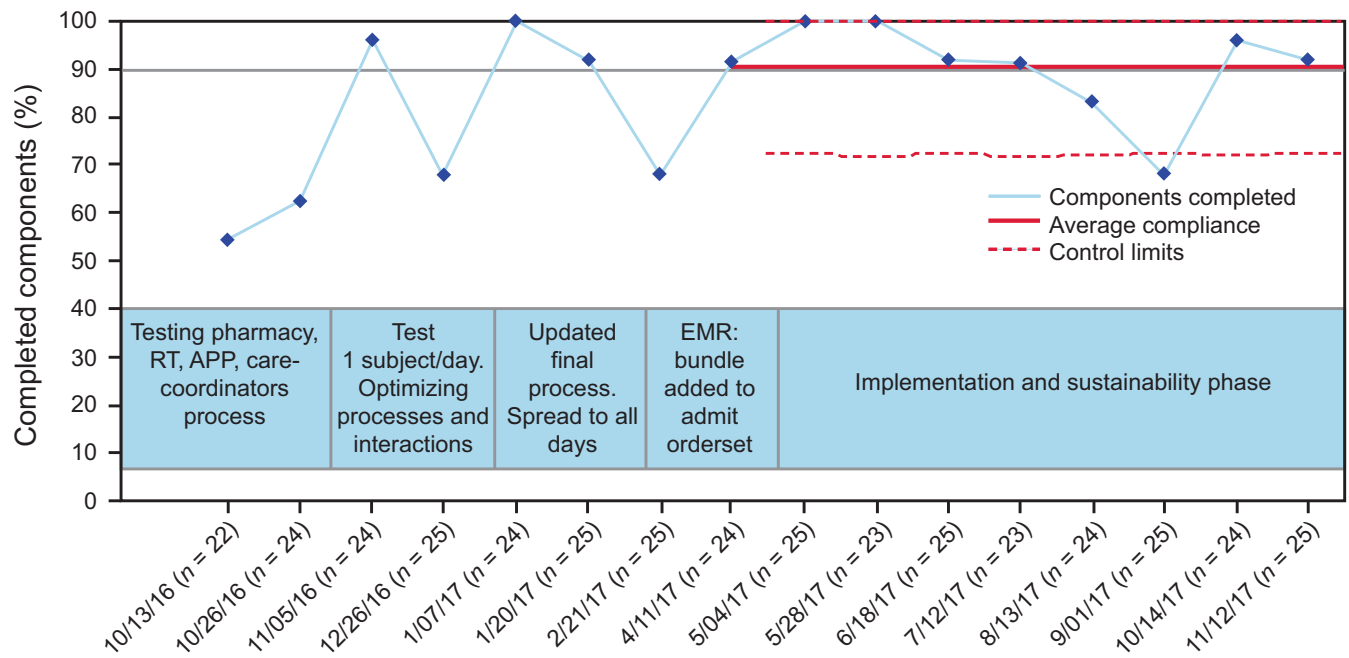

Time series with 5 subjects/cycle (date of last subject encounter)

Fig. 2. Adherence to the COPD care bundle components among subjects with COPD exacerbation who were discharged from ED-Obs (process measure) is displayed on a statistical process control P-chart. The time-series on the $x$ axis is based on 5 consecutive patients discharged to home from ED-Obs. The date on the $x$ axis signifies the last encounter date with the subject. Every applicable bundle component was defined as an opportunity. The percent of applicable bundle components that were completed is displayed on the $y$ axis. The goal was set for $>90 \%$ adherence (gray line). The phases of plan-do-study-act cycles and implementation are described. Because this was a new process, the control limits and average adherence was calculated once we reached the implementation and sustainability phase. ED-Obs $=$ emergency department observation unit; $\mathrm{RT}=$ respiratory therapist; $\mathrm{APP}=$ advance practice provider; $\mathrm{EMR}=$ electronic medical record.

incorporation of the COPD care bundle order set as part of the ED observation unit admission orders for subjects with COPD exacerbation, and a discharge checklist to ensure bundle components were completed before patient discharge. The COPD care bundle EMR order set activates all team members (ie, RT, community health worker, pharmacy, and care coordinator) for their respective roles and facilitates prescription of appropriate COPD medications for discharge. The final process map with roles of team members and levels of process reliability is shown in Figure 1. Adherence of COPD care bundle components increased over the 6 months of testing and has been maintained at $90 \%$ on average since the implementation of the final process (Fig. 2).

\section{Outcome Measures}

Among those discharged home, the rate of 30-d allcause ED revisit after bundle implementation was 30\% with a special cause system shift on SPC. This represents a $38.6 \%$ relative reduction compared to the baseline period $(48.9 \%$ vs $30 \%, P=.003$ ) (Fig. 3, Table 2). During the testing and implementation phase, there were subjects who did not receive all the bundle components. Among the subjects who received 4 or 5 bundle components, the revisit rate was $26.6 \%(n=62)$, whereas those who received $\leq 3$ bundle components had a revisit rate of 44.4\% $(n=18)(P=.12)$.

\section{Balancing Measure (Hospitalization Rate Before and After Intervention)}

During the 13-month period after bundle implementation (October 2016 through November 2017), a total of 165 subjects with COPD exacerbation were admitted to the ED observation unit; $85(51.5 \%)$ of these subjects were hospitalized, and $80(48.5 \%)$ were discharged to home at the end of their stay in the ED observation unit. Neither the rate of admission of subjects with COPD exacerbations to the ED observation unit (15.8 vs 12.7 admissions/month, pre vs post) nor the rate of hospitalization from the ED observation unit (balancing measure) changed compared to baseline period $(P=.16)$.

\section{Discussion}

In this quasi-experimental single-center study, reliable implementation of a 5-component COPD care bundle in the ED observation unit led to a significant reduction in 30-d all-cause ED revisits among subjects with COPD exacerbations. A $38.6 \%$ relative reduction (from $48.9 \%$ to $30 \%$ ) was observed with a trend toward higher reduction among subjects who received all 5 components of the bundle. Using improvement science methodology, the project team was able to implement a new care-delivery process in the ED observation unit designed to achieve $90 \%$ reliable adherence to a COPD care bundle. Subject characteristics and 


\section{COPD CARE BUNDLE IN THE EMERGENCY DEPARTMENT}

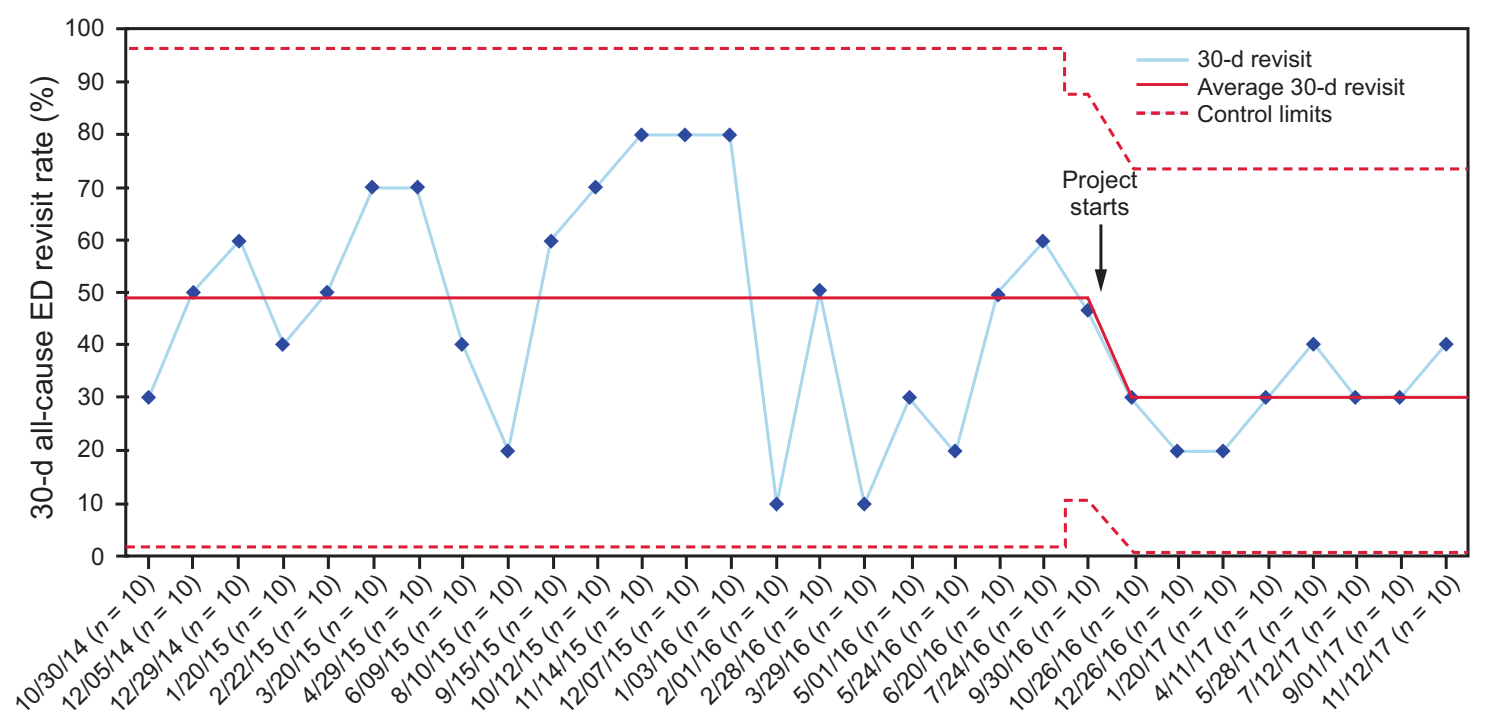

Time series with 10 subjects/cycle (date of last subject encounter)

Fig. 3. 30-d all-cause ED revisit rate among subjects with COPD exacerbation who were discharged from ED-Obs (outcome measure) is displayed on a statistical process control P-chart. Due to month-to-month variation in the number of patients discharged from ED-Obs, the time series on the $x$ axis is based on every 10 consecutive patients. The dates on the $x$ axis signify the date of last encounter with the subject. The 30-d all-cause ED revisit rate (\%) is on the $y$ axis. The baseline period extends from August 2014 through September 2016, showing a stable system with common cause (random) variation only. The last subgroup in the baseline period has 15 patients, after which the bundle testing began in August 2016. During the postbundle period, the data show a system-shift with 8 consecutive observations below the center-line (49\%). New control limits were hence calculated with a new average of $30 \%$. ED-Obs = emergency department observation unit.

Table 2. Rates of Hospitalization and 30-d All-Cause Revisits Among COPD Exacerbation Subjects in ED-Obs

\begin{tabular}{|c|c|c|c|c|c|c|}
\hline & $\begin{array}{l}\text { Baseline Period } \\
\qquad(26 \mathrm{mo})\end{array}$ & $\begin{array}{l}\text { Postbundle Period } \\
\qquad(13 \mathrm{mo})\end{array}$ & $P\left(\chi^{2}\right)$ & $\begin{array}{l}\text { Baseline } \\
\text { Sample }\end{array}$ & $\begin{array}{l}\text { Postbundle } \\
\text { Sample }\end{array}$ & $P\left(\chi^{2}\right)$ \\
\hline Subjects admitted to ED-Obs & 410 & 165 & & 50 & 83 & \\
\hline \multicolumn{7}{|l|}{ Disposition from ED-Obs } \\
\hline Hospitalization & $185(45.1)$ & $85(51.5)$ & .16 & $22(44)$ & $40(48)$ & .64 \\
\hline Home discharge & $225(54.9)$ & $80(48.5)$ & .16 & $28(56)$ & $43(52)$ & .64 \\
\hline 30-d revisit among discharged & $110(48.9)$ & $24(30)$ & .003 & $14(50)$ & $11(25.6)$ & .035 \\
\hline
\end{tabular}

Data are presented as $n(\%) . P<.05$ considered significant.

ED-Obs $=$ emergency department observation unit

hospitalization rate from ED observation unit were similar in the before- and the after-bundle periods, suggesting that the reduction in the all-cause $30-\mathrm{d}$ revisit rate is related to the implementation of the COPD care bundle.

There is an urgent need to improve outcomes and reduce cost of care for COPD exacerbations, but there is a paucity of evidence on best practices in ED observation units that can lead to this improvement. ${ }^{31}$ Protocols and care-delivery processes for managing subjects with COPD exacerbations in the ED observation unit setting are adapted from in-patient settings and published guidelines, which may be limited in their applicability to this unique observation setting. ${ }^{32}$ One study reported that subjects with a COPD exacerbation who were cared for in an ED observation unit compared to in-patient admission had shorter lengths of stay and lower mortality, but higher rates of $10-\mathrm{d}$ readmission. ${ }^{33}$

To our knowledge, this is the first study to report the effectiveness of a COPD care bundle implemented in an ED observation unit with a direct impact on 30-d all-cause ED revisits. COPD care bundles have commonly been studied among hospitalized patients with variable success. The differences in success relate to variations in bundle components and often poor adherence to the bundle. ${ }^{21}$ The success of the COPD care bundle used in this study is likely due to multiple factors: it was constructed with a comprehensive understanding of local care-delivery failures and identification of unmet patient needs, ${ }^{20}$ the bundle had a track record 


\section{COPD CARe Bundle In the EMERGENCy DePartment}

of success in the in-patient setting for reducing hospital readmissions,${ }^{20}$ and the process was reliably implemented in routine care delivery with $90 \%$ adherence after multiple iterative PDSA cycles and using implementation science methodologies.

One pragmatic challenge in health care is to translate the ideal state of a research protocol into routine work. In this article, in addition to reporting the impact of this COPD care bundle on patient outcomes, we elaborate the context in which the team implemented this bundle. The newly designed workflow and the tools utilized are shared explicitly to facilitate rapid and easy adoption in other systems within their unique contexts. The resources available and the roles of individual team members may differ for each facility. For example, we were fortunate in that we did not have to expand hours or add staff to implement this COPD bundle. Through small, focused testing cycles, we were able to identify learning opportunities, celebrate early successes, and foster continued engagement with the project's goal. Because of previous institutional experience with the in-patient COPD bundle and ability to demonstrate its success, bundle implementation in the ED setting was welcomed by ED staff and familiar to many personnel involved, specifically the RTs. We also recommend that hospitals evaluate their local failures and consider additions to the current COPD care bundle depending on their specific needs. A strength of our study is that it included all subjects who were deemed to have COPD exacerbations by clinical assessment and ICD coding. Hence, the change in outcome is reflective of real-life conditions and clinical scenarios, which makes it more replicable.

Our study has several limitations. It is a single-center study at an academic, safety-net, tertiary care hospital representing a relatively sick population with high baseline case-mix index of 2.12, high baseline ED revisit rate, and local burden of disease. Therefore, the impact on outcomes may vary at other institutions due to regional and contextual differences. ${ }^{34}$ Additionally, although we demonstrate the effectiveness of the bundle as a whole and observed a dose effect in outcomes, the study design did not allow for analyzing the impact of individual bundle components on the outcome measure. Rather, all bundle components were selected based on supporting literature and contextual relevance to mitigate care-delivery failures and unmet patient needs. Because this was a novel implementation of a caredelivery process, not every subject completed all 5 components of the bundle each time (Fig. 2). This was due to a variety of factors, including subject refusal of personalized inhaler education, leaving before a follow-up appointment was scheduled, staff shortages causing delayed inhaler education, and 30-d inhaler supply already available to the subject. Another limitation of our study design is that we did not have a concurrent control population for comparison of factors other than the bundle. However, our baseline and postbundle patient samples demonstrate similar characteristics. The baseline subjects seemed to have higher comorbidity scores, whereas the postbundle subjects had lower percent of predicted $\mathrm{FEV}_{1}$, both of which increase the risk of ED revisit. ${ }^{35,36}$ Lastly, the revisit rates are assessed by individual encounters rather than by individual subjects. Frequent ED utilization is common among individual subjects with COPD, and some subjects had more than one encounter in our system throughout the study period. We did not observe any difference in prevalence of high users (subjects with $\geq 3$ or $\geq 5$ ED visits/year) in the postbundle period (unpublished observations). Improving outcomes for this subset of COPD patients will require focused interventions that address comorbid conditions and socioeconomic factors that were beyond the scope of our intervention.

Because improvement is a continuous journey, we will sustain reliable adherence to the COPD care bundle while further exploring innovative approaches to ED decision making regarding disposition and level of care in this vulnerable patient population.

\section{Conclusions}

Effective and pragmatic interventions are needed to improve outcomes of patients with COPD exacerbation who are managed in ED observation units. Reliable adherence to a COPD care bundle, designed with contextual knowledge of care-delivery failures and unmet patient needs, reduced 30-d all-cause ED revisits among subjects with COPD exacerbations treated in our ED observation unit.

\section{ACKNOWLEDGMENTS}

The authors thank Jessica Winter PharmD, Nicole Harger PharmD, Michele Long, Maureen Lauren RN, and Francisco J Fernandez MD MSc.

\section{REFERENCES}

1. Ford ES, Croft JB, Mannino DM, Wheaton AG, Zhang X, Giles WH. COPD surveillance - United States, 1999-2011. Chest 2013;144(1): 284-305.

2. Donaldson GC, Seemungal TAR, Bhowmik A, Wedzicha JA. Relationship between exacerbation frequency and lung function decline in chronic obstructive pulmonary disease. Thorax 2002;57(10):847-852.

3. Seemungal TAR, Donaldson GC, Paul EA, Bestall JC, Jeffries DJ, Wedzicha JA. Effect of exacerbation on quality of life in patients with chronic obstructive pulmonary disease. Am J Respir Crit Care Med 1998;157(5 Pt 1):1418-1422.

4. Suissa S, Dell'Aniello S, Ernst P. Long-term natural history of chronic obstructive pulmonary disease: severe exacerbations and mortality. Thorax 2012;67(11):957-963.

5. Toy EL, Gallagher KF, Stanley EL, Swensen AR, Duh MS. The economic impact of exacerbations of chronic obstructive pulmonary disease and exacerbation definition: a review. COPD 2010;7(3):214-228.

6. Vestbo J, Edwards LD, Scanlon PD, Yates JC, Agusti A, Bakke P, et al. Changes in forced expiratory volume in 1 second over time in COPD. N Engl J Med 2011;365(13):1184-1192. 


\section{COPD CARE BundLE IN THE EMERGENCY DEPARTMENT}

7. Strassels SA, Smith DH, Sullivan SD, Mahajan PS. The costs of treating COPD in the United States. Chest 2001;119(2):344-352.

8. Centers for Medicare and Medicaid Services. Hospital value-based purchasing. Available at: https://www.cms.gov/medicare/qualityinitiatives-patient-assessment-instruments/hospitalqualityinits/hospitalvalue-based-purchasing.html. Accessed December 2, 2019.

9. Centers for Medicare and Medicaid Services. Readmissions reduction program (HRRP). Available at: https://www.cms.gov/medicare/ medicare-fee-for-service-payment/acuteinpatientpps/readmissionsreduction-program.html. Accessed December 2, 2019.

10. García-Sanz MT, Pol-Balado C, Abellás C, Carlos Cánive-Gómez JC, Antón-Sanmartin D, González-Barcala FJ. Factors associated with hospital admission in patients reaching the emergency department with COPD exacerbation. Multidiscip Respir Med 2012;7(1):6.

11. Hasegawa K, Tsugawa Y, Tsai C-L, Brown DFM, Camargo CA. Frequent utilization of the emergency department for acute exacerbation of chronic obstructive pulmonary disease. Respir Res 2014;15 (1):1-13.

12. Rowe BH, Villa-Roel C, Guttman A, Ross S, Mackey D, Sivilotti MLA, et al. Predictors of hospital admission for chronic obstructive pulmonary disease exacerbations in Canadian emergency departments. Acad Emerg Med 2009;16(4):316-324.

13. Stiell IG, Clement CM, Aaron SD, Rowe BH, Perry JJ, Brison RJ, et al. Clinical characteristics associated with adverse events in patients with exacerbation of chronic obstructive pulmonary disease: a prospective cohort study. CMAJ 2014;186(6):E193-E204.

14. Tsai CL, Clark S, Cydulka RK, Rowe BH, Camargo CA. Factors associated with hospital admission among emergency department patients with chronic obstructive pulmonary disease exacerbation. Acad Emerg Med 2007;14(1):6-14.

15. Shah T, Press VG, Huisingh-Scheetz M, White SR. COPD readmissions: addressing COPD in the era of value-based health care. Chest 2016;150(4):916-926.

16. Lindenauer P, Pekow P, Gao S, Crawford AS, Gutierrez B, Benjamin E. Quality of care for patients hospitalized for acute exacerbations of chronic obstructive pulmonary disease. Ann Intern Med 2006;144 (12):894-903.

17. Auerbach AD, Kripalani S, Vasilevskis EE, Sehgal N, Lindenauer PK, Metlay JP, et al. Preventability and causes of readmissions in a national cohort of general medicine patients. JAMA Intern Med 2016;176(4):484-493.

18. Quintana JM, Unzurrunzaga A, Garcia-Gutierrez S, Gonzalez N, Lafuente I, Bare M, et al. Predictors of hospital length of stay in patients with exacerbations of COPD: a cohort study. J Gen Intern Med 2015;30(6):824-831.

19. Krumholz HM, Wang K, Lin Z, Dharmarajan K, Horwitz LI, Ross JS, et al. Hospital-readmission risk - isolating hospital effects from patient effects. N Engl J Med 2017;377(11):1055-1064.

20. Zafar MA, Panos RJ, Ko J, Otten LC, Gentene A, Guido M, et al. Reliable adherence to a COPD care bundle mitigates system-level failures and reduces COPD readmissions: a system redesign using improvement science. BMJ Qual Saf 2017;26(11):908-918.

21. Ospina MB, Mrklas K, Deuchar L, Rowe BH, Leigh R, Bhutani M, Stickland MK. A systematic review of the effectiveness of discharge care bundles for patients with COPD. Thorax 2017;72:31-39.
22. Vogelmeier CF, Criner GJ, Martinez FJ, Anzueto A, Barnes PJ, Bourbeau J, et al. Global strategy for the diagnosis, management, and prevention of chronic obstructive lung disease 2017 report. GOLD executive summary. Am J Respir Crit Care Med 2017;195(5):557-582.

23. Blee J, Roux RK, Gautreaux S, Sherer JT, Garey KW. Dispensing inhalers to patients with chronic obstructive pulmonary disease on hospital discharge: effects on prescription filling and readmission. Am J Health Syst Pharm 2015;72(14):1204-1208.

24. Zwerink M, Brusse-Keizer M, van der Valk PD, Zielhuis GA, Monninkhof EM, van der Palen J, et al. Self-management for patients with chronic obstructive pulmonary disease. Cochrane Database Syst Rev 2014(3):CD002990.

25. Charlson ME, Pompei P, Ales KL, MacKenzie CR. A new method of classifying prognostic comorbidity in longitudinal studies: development and validation. J Chronic Dis 1987;40(5):373-383.

26. Langley G, Moen R, Nolan K, Nolan T, Norman C, Provost L. Methods for improvement. In: The improvement guide: a practical approach to enhancing organizational performance, 2nd ed. San Francisco: Jossey-Bass; 2009:73-262.

27. Nolan T, Resar R, Haraden C, Griffin FA. Improving the reliability of health care. IHI Innovation Series white paper. Boston: Institute for Healthcare Improvement; 2004

28. Benneyan JC, Lloyd RC, Plsek PE. Statistical process control as a tool for research and healthcare improvement. Qual Saf Health Care 2003;12(6):458-464.

29. Amin S. Control charts 101: a guide to health care applications. Qual Manag Health Care 2001;9(3):1-27.

30. Perla RJ, Provost LP, Murray SK. Sampling considerations for health care improvement. Qual Manag Health Care 2014;23(4):268-279.

31. Baugh C, Mace S, Pena M. The evidence basis for observation care for adults based on diagnosis/clinical condition. In: Mace SE, editor. Observation medicine principles and protocols. Cambridge: Cambridge University Press; 2017:430-477.

32. Pena ME, Kazan VM, Helmreich MN, Mace SE. Care of respiratory conditions in an observation unit. Emerg Med Clin North Am 2017;35 (3):625-645.

33. Salazar A, Juan A, Ballbe R, Corbella X. Emergency short-stay unit as an effective alternative to in-hospital admission for acute chronic obstructive pulmonary disease exacerbation. Am J Emerg Med 2007;25 (4):486-487.

34. Croft JB, Wheaton AG, Liu Y, Xu F, Lu H, Matthews KA, et al Urban-rural county and state differences in chronic obstructive pulmonary disease - United States, 2015. MMWR Morb Mortal Wkly Rep 2018;67(7):205-211.

35. Spece LJ, Epler EM, Donovan LM, Griffith MF, Collins MP, Feemster LC, et al. Role of comorbidities in treatment and outcomes after chronic obstructive pulmonary disease exacerbations. Ann Am Thorac Soc 2018;15(9): 1033-1038.

36. Quintana JM, Esteban C, Garcia-Gutierrez S, Aguirre U, Gonzalez N, Lafuente I, et al. Predictors of hospital admission two months after emergency department evaluation of COPD exacerbation. Respiration 2014;88(4):298-306.

This article is approved for Continuing Respiratory Care Education credit. For information and to obtain your CRCE

(free to AARC members) visit

www.rcjournal.com

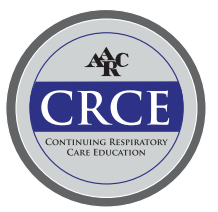

